\title{
An Effective Reasoning Algorithm for Question Answering System
}

\author{
Poonam Tanwar ${ }^{1}$, \\ ${ }^{1}$ Asst. Professor, Dept of CSE, \\ Lingaya's University, Faridabad, \\ Haryana, India \& PhD Scholar, \\ Uttarakhand Technical University, \\ Dehradun,India
}

\author{
Dr. T. V. Prasad ${ }^{2}$, \\ ${ }^{2}$ Former Dean of Computing \\ Sciences, Visvodaya Technical \\ Academy, Kavali, AP, \\ India.
}

\author{
Dr. Kamlesh Datta ${ }^{3}$ \\ ${ }^{3}$ Assoc. Prof \& HOD (CSE), \\ National Institute of Technology, \\ Hamirpur, Himachal Pradesh, \\ India
}

\begin{abstract}
Knowledge representation (KR) is the most desirable area of research to make the system intelligent. Today is the era of knowledge that requires articulations, semantic, syntax etc. These requirements, forced to design a general system which is applicable to represent declarative as well as procedural knowledge. Without effective inference/reasoning mechanism, the strength and utility of knowledge representation technique fulfill the partial requirement for an intelligent system. The objective of this research work is to present the effective/ appropriate knowledge representation technique for representing the general knowledge and a reasoning algorithm for Question Answering system (QAS) work as story reader, so that appropriate knowledge can be infer from the system. The architecture of knowledge representation system is capable to integrate different type of knowledge and it is cost effective also.
\end{abstract}

Keywords-Knowledge Representation (KR); Semantic Net; Script; Reasoning; QAS; NLP

\section{INTRODUCTION}

AI is the branch of science to make the machine as intelligent as human being for particular domain. Alternatively, it is the study of making machine intelligent by implementing intelligent programs to perform the complicated task. In 1950s, Alan Turing presented a paper on Computing Machinery and Intelligence. The result of this paper was if a machine could pass certain test (known as Turing test) then it could be intelligent. In this paper Turing also considered a number of arguments for, and objections to, the idea that computers could exhibit intelligence [1].

Knowledge representation (KR) is an essential area for cognitive science and Artificial Intelligence. In former, it is concerned with how knowledge is stored and processed, while in the latter the main aim is to solve problems requiring intelligence which otherwise is possible only through knowledge.

Broadly, KR is a study of methods of how knowledge is actually visualized/ realized and how efficiently/naturally it is similar to the depiction of knowledge in human brain. Constructing intelligent systems require large amount of knowledge and a method for representing large amounts of knowledge that permits interaction [1] [3]. $\mathrm{KR}$ is the fundamental issue in AI that attempt to understand intelligence [1] [2].
The main problem of AI system is how to represent knowledge and how to incorporate both types of knowledge in single system i.e., declarative and procedural [1]. Due to these issues, KR became a separate research area in AI. Since last few years a group of (two or more) methods are being considered as hybrid KR system that can address all these fundamental issues. KR techniques can be used for representing the knowledge required for Question Answering system.

\section{a) KR Techniques}

The KR techniques are divided in many categories. The representation techniques can be declarative, procedural, hierarchical, graphical, etc. Objects, properties, [17] categories and relations between objects, situations, events, states and time [17], causes and effects are the things that an intelligent system desires to represents [4][8]. The semantic net, conceptual dependency and script KR technique are described here. Semantic Net is commonly used KR technique that represents the connection between objects or class of objects. It is a directed graph in which nodes / vertices represent the objects/ class of objects and edges and links (unidirectional) represent the semantic relations between the objects. Semantic net are used to represent the inheritable knowledge. Inheritance is most useful form of inference. Inheritance is the property in which element of some class inherit the attribute and values from some other class [5][6][9].

The variant of semantic net i.e., partitioned semantic net can be used to delimit the scopes of quantified variables. [6][9].CD was developed by Roger Schank in 1973 to represent the knowledge acquired from natural language input. In CD Sentences are represented as a series of diagrams depicting actions using the abstract and real physical situations. CD representation provides the sets of primitive actions, different types of states, and different theories of inference. A variation in the theme of structured objects called scripts was devised by Roger Schank and his associates in 1973[5] [9][10]. Frame KR technique is also widely used based on object oriented concept. Many hybrid KR techniques also came for getting the advantage of KR techniques KL-ONE KR tool was the first hybrid KR technique which is the hybrid of semantic net and first order predicate logic. The FRORL, RT-FRORL are hybrid KR techniques [4][12][17[20]. In SOL, Hybrid KR the concept of smart object was used with encapsulation. 


\section{b) Question Answering System}

An artificial QAS can be made for various applications like search engine, Natural language processing, Machine learning. This section presents a survey of various QAS.

i) CNLP AIDE: It is a off line system, based on four modules, question-answering system, document processing, Language-to-Logic (L2L), Search Engine, and Answer Providing Passages. Document processing is done offline, shown in figure 1 . When a question is submitted by the user, it directly sent to the Language-toLogic module, that generates the L2L query representation. The Search Engine module then searches the index and returns the top 200 relevant passages [21].

ii) TREC-9: It was the extension of TREC-8 and was based on a combination of the Okapi retrieval engine, Microsoft's natural language processing system and a module for matching logical forms. The questions was analyzed by NLPWin to produce a logical form. The query was contain the words like what, who, how, etc and stop words. Okapi IR engine was used for query term; the BM25 was used to retrieve weighting list of documents. The documents were segmented into sentences architecture of the system, Figure 2 [22].

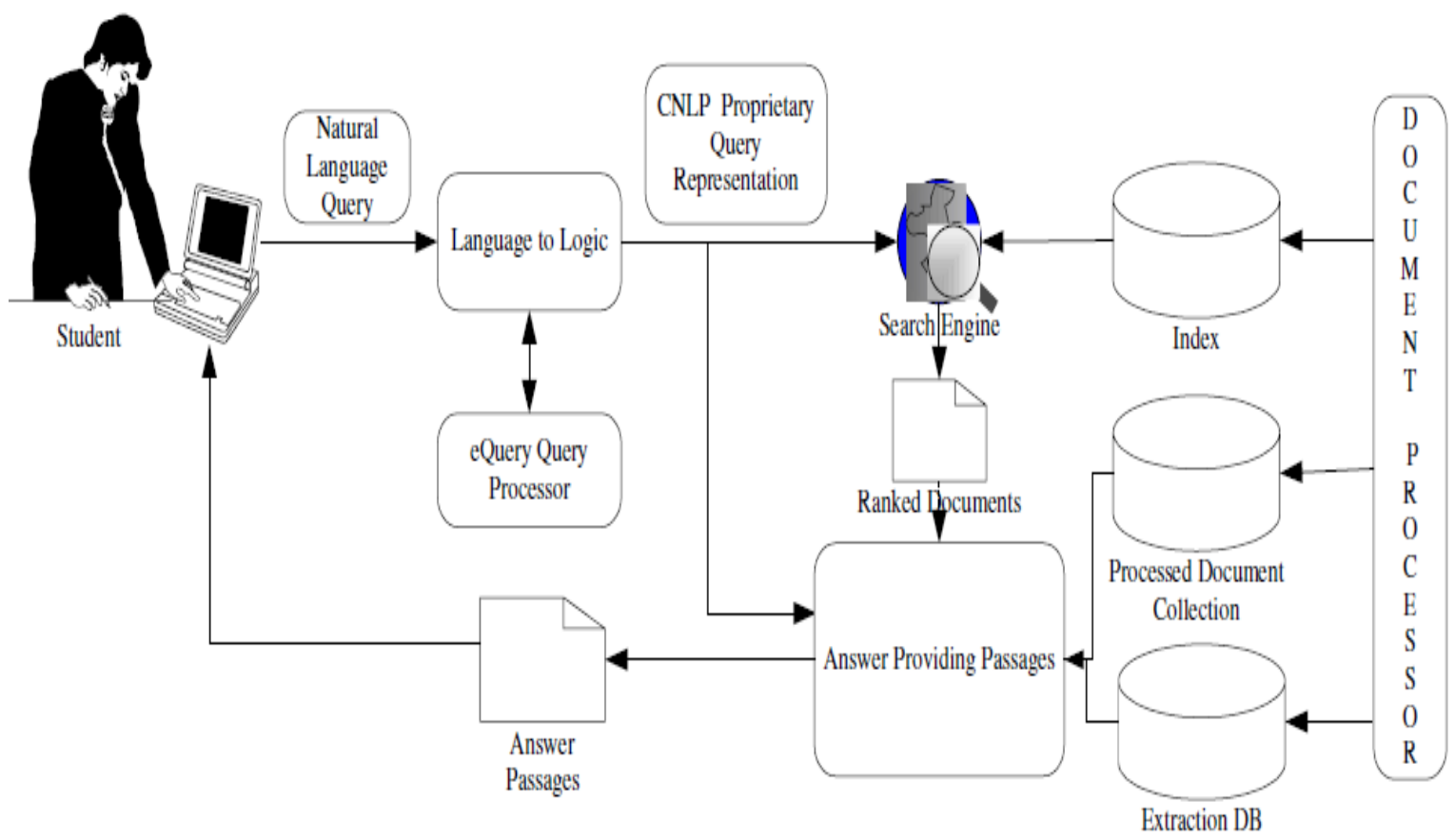

Fig. 1. Architecture of CNLP AIDE [21]

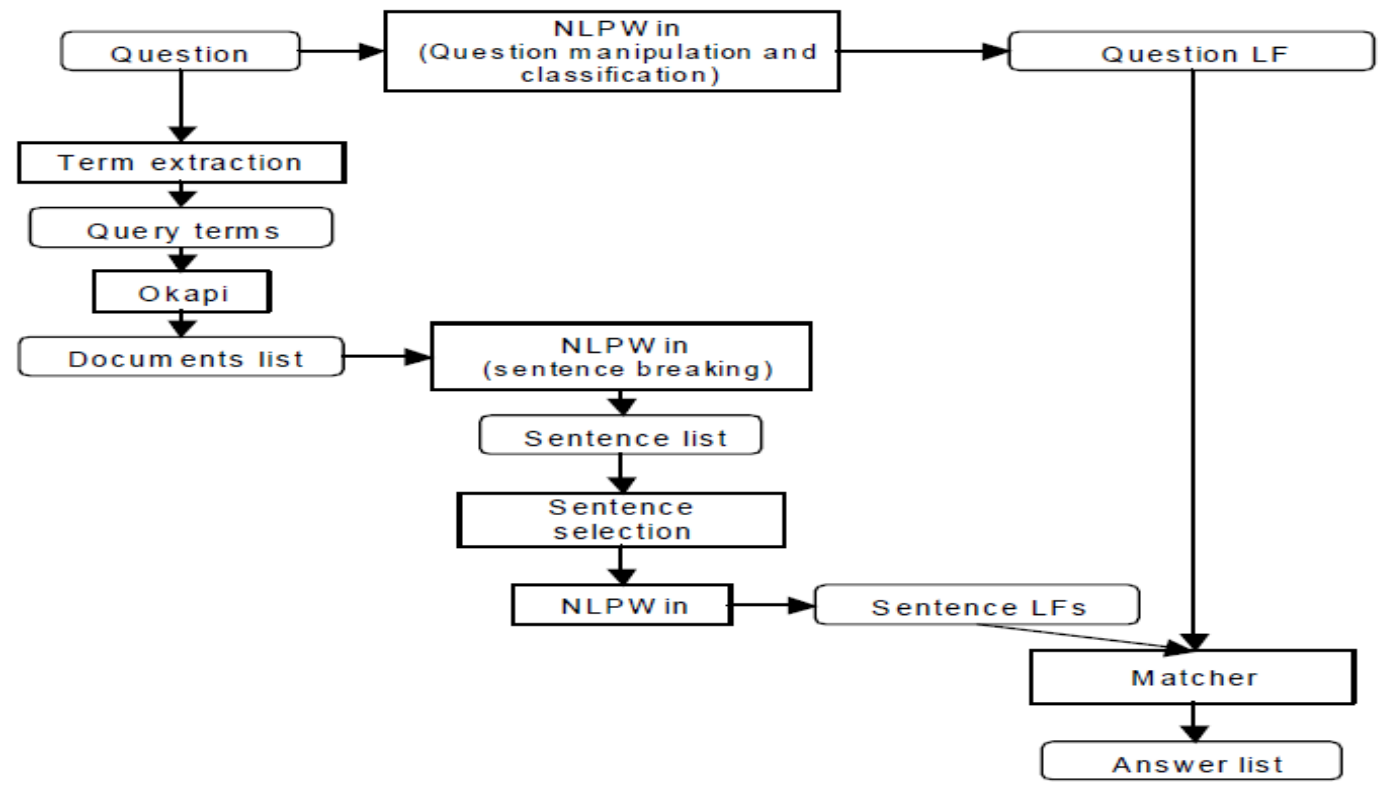

Fig. 2. Architecture of the TREC-9 [22] 
c) IQAS: It was proposed in year 2011 with architecture as in Figure 3. IQAS was designed to help the students so that they could become the good reader, it process the input in NLP and provide the result with proper feedback. The performance of IQAS is based on the no of documents, the information that user need and relevance judgments [23].

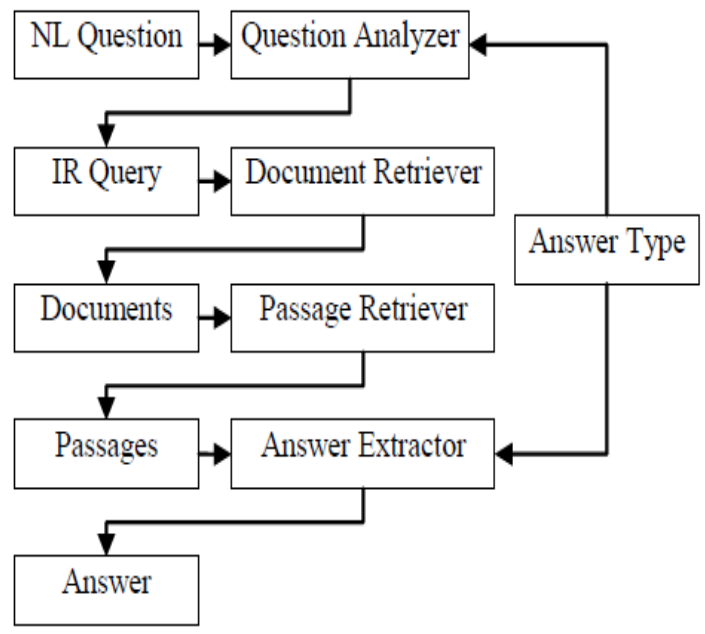

Fig. 3. represents the architecture of IQAS [23]

\section{KnOwledge Base System Architecture}

The research in AI is divided in to two categories $\mathrm{KR}$ and general. For making the computer or machine as intelligent as human being, it requires two things $\mathrm{KR}$ and inference mechanism. Development of an AI system is a crucial task because some time we have incomplete information and it can be ambiguous and uncertain. So solution to these problems is to build a knowledge effective knowledge base and an effective inference mechanism.

\section{a) Knowledge Base (KB)}

The knowledge base used in Figure 4 and Figure 7 is used to store the knowledge required to solve the problem domain. The KB in Figure 4 is used to store the incoming knowledge i.e., story and the hybrid representation corresponding to that story whereas the KB used in Figure 6 is used to store the rules required to inference the knowledge from the input.

The KR system must be able to represent any type of knowledge, “a) syntactic, b) semantic, c) logical, d) presupposition, e) understanding ill formed input, f) ellipsis, g) case constraints, and h) vagueness". For making it more effective the knowledge representation model is divided in to five sub parts the $\mathrm{K}$ Box, knowledge base, query applier, reasoning and user interface as shown in Figure 4.[3][8].

The knowledge base architecture defined in Figure 4 is used as a story reader. The knowledge base of the system is capable to store the knowledge which is a hybrid of semantic net and script. Semantic net is used to represent the inheritable and relational knowledge whereas semantic net is used to represent the events in the story shown in Figure 5. The methodology used to implement the system is given in Figure 6.

The source of input can to the system can be a book, newspaper, magazine etc. a check is made whenever a new input is entered by the user to see whether the same is already stored in knowledge base or not. If the same is already stored in knowledge base then system displays the alert message or else it accepts the new input and passes on for further processing. As shown in Figure 5 the system is able to take the input from outside word.

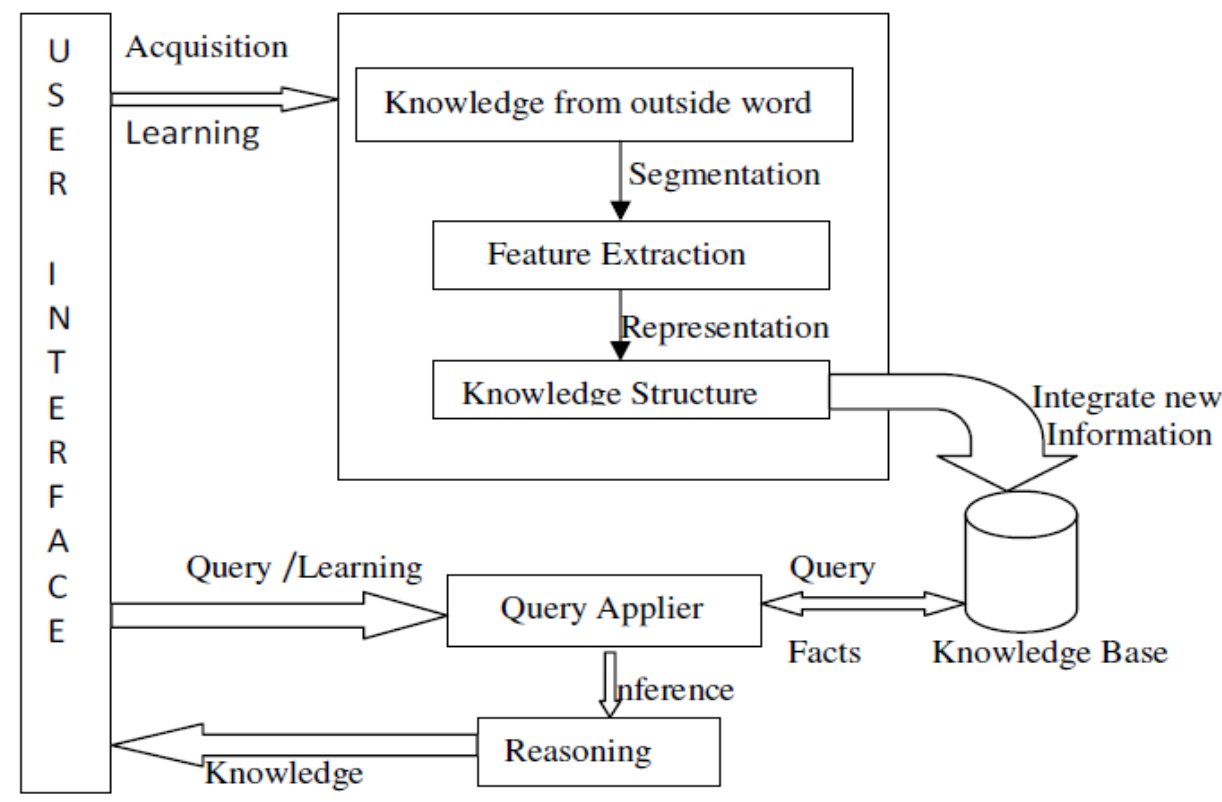

Fig. 4. Knowledge Base System Model / Architecture [3][8]. 

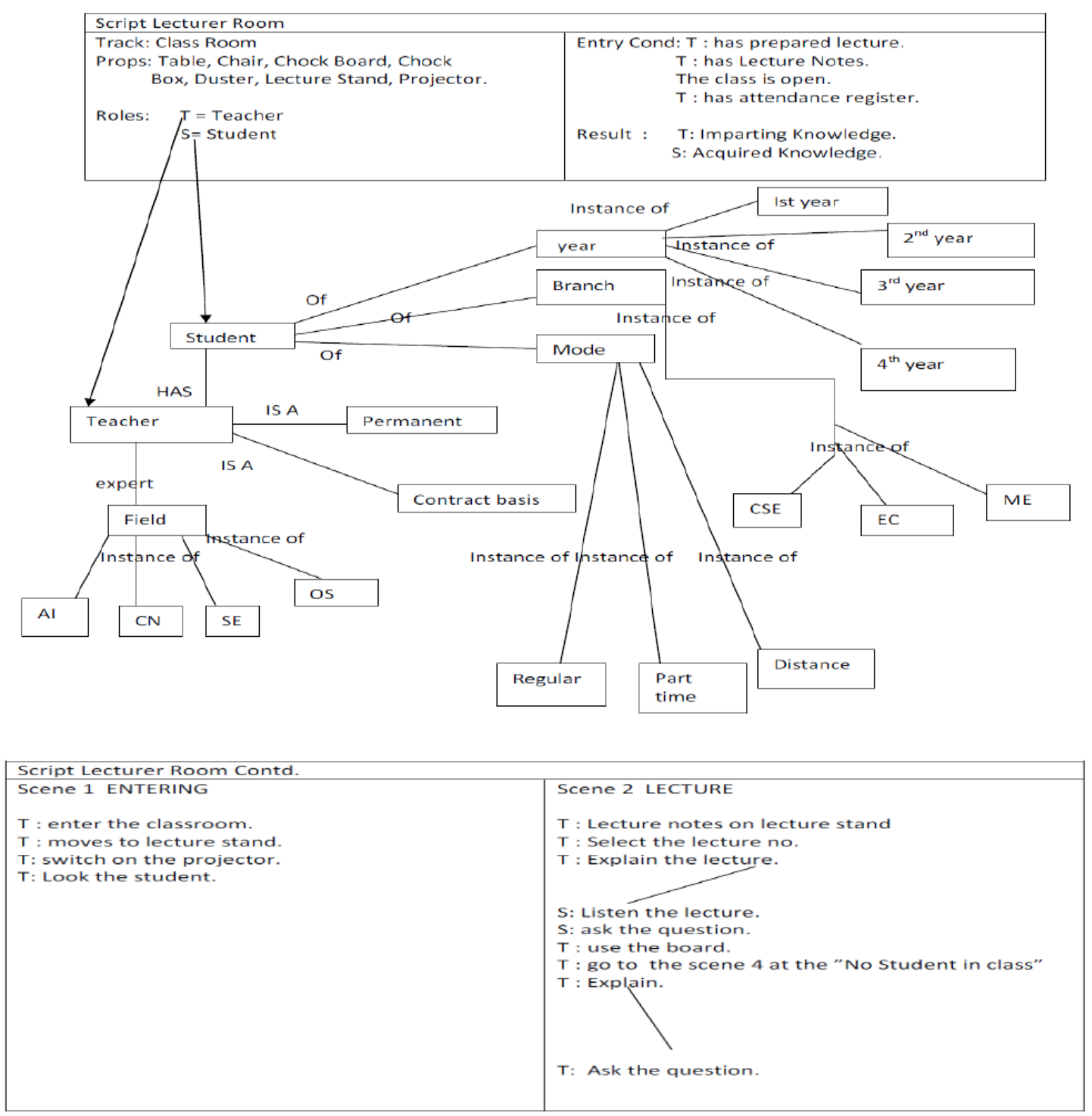

Fig. 5. Logical view of Hybrid KR [3][8].

\section{b) Hybrid KR}

The Hybrid KR used was the hybrid of semantic net and script KR techniques. The logical structure is shown in Figure 5 . The system generates the semantic net for each input and links the semantic net with script of the story. The Hybrid KR was used to visualize the knowledge required for class room as well as the knowledge about students. For example, if any one wants to know the details of students whether he/she is 2nd year student and currently studying in semester 4 then the system can represent this aspect using semantic net and to visualise the interconnections that took place in the context of the class room, semantic net must be used. After generating the hybrid structure, a better detail will be available as to when the student will finish his/her degree. This hybrid structure proved to be very efficient in any situation where inheritable as well as stereotype knowledge was required.

\section{c) Query Applier}

Query Applier is used for obtaining the facts from the system and then passes the knowledge to the inference mechanism for reasoning [7]. Whenever the new query comes from the system will learn whether that query is related to the previous query or it generates from the previous query and check how many times users ask the combination of these [7]. We have used the association learning rule mining in the system for making the system exhibit characteristics of being intelligent. 


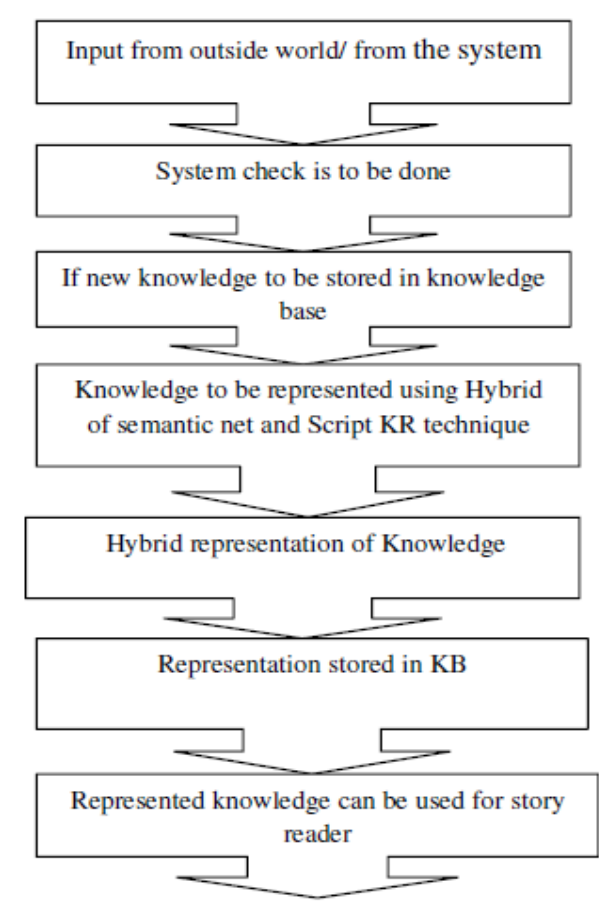

Fig. 6. Methodology of the KB

\section{d) Reasoning Algorithm}

Reasoning [24] system is used for getting new fact from the existing knowledge or to draw inference for the situation. The inference can be inductive / deductive. There are many algorithms available for searching the goal and are divided in to two categories i.e. a) uninformed search techniques (depth first and breadth first) and $b$ ) heuristic/informed search techniques (best first search, $\mathrm{A}^{*}, \mathrm{AO}^{*}$, etc.) in AI. Resolution and chaining (forward and backward) are the known reasoning techniques. Forward chaining refers to deduction whereas the backward chaining refers to induction. An example is considered here for deductive and inductive reasoning. The example of former is "Poonam must be either cooking or washing clothes". If she is not cooking she must be washing clothes. i.e., in case of deductive reasoning the truth of premises must leads to truth of conclusion. The example of inductive i.e., is "the initial failure of machine was caused by some spare part failure". i.e., the truth of premise supports the conclusion without giving exact assurance.

In forward chaining each statement is act as premises and each rule is divided to two parts the left side and the right side. The left side of the rule is being used to match with current condition. If the current condition matches with any or more than one rules on the left side then the right side of the matched rules are applied as the action to be performed. In the same way, system starts from the initial premises and applies forward chaining and moves towards the goal i.e., the conclusion of the given knowledge.

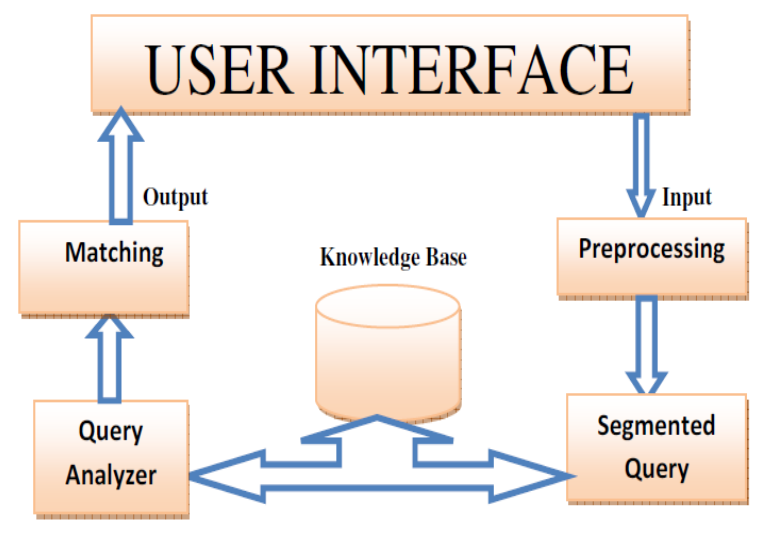

Fig. 7. Architecture of an artificial story reader System as QAS.

The system is able to adequately provide answer for any query related to the input using forward reasoning. Since every problem is unique in nature and has its own complications/ difficulties, the problem domain will require extensive and unique set of knowledge to solve the identified problem. AI requires a collection of knowledge and control mechanism to resolve a specific problem in a systematic fashion to yield the result to satisfaction. below.

Algorithm used by the system for query applier is given

Algorithm Forward (KR, KB, K): returns a substitute that was not found in $\mathrm{K}$.

1. Repeat until KB is empty or NEW is not found.

2. Initialize NEW

3. $\mathrm{NEW} \leftarrow\{\varepsilon\}$

4. For each statement $\mathrm{S}$ in $\mathrm{KB}$ do.

5. $\left(\mathrm{S} 1{ }^{\wedge} \mathrm{S} 2{ }^{\wedge} \mathrm{S} 3^{\wedge}---\Rightarrow \mathrm{Q}\right) \leftarrow(\mathrm{A})$

6. Term $\leftarrow \lambda$

7. For each $\lambda$ in $\mathrm{Q}$ : such that $\left(\mathrm{S} 1{ }^{\wedge} \mathrm{S} 2{ }^{\wedge} \mathrm{S} 3\right) \lambda=\left(\mathrm{S} 1^{\prime} \wedge \mathrm{S} 2^{\prime}\right.$

$\left.{ }^{\wedge} \mathrm{S} 3^{\prime}\right) \lambda$ in story for some $\mathrm{S1}^{\prime}, \mathrm{S} 2^{\prime}, \mathrm{S} 3^{\prime}$--- in KB.

8. $\mathrm{Q}^{\prime} \leftarrow(\lambda, \mathrm{Q})$

If $\mathrm{Q}^{\prime}$ not in query then

9. $\mathrm{Q}^{\prime} \leftarrow \mathrm{NEW}$

10. Add $\mathrm{Q}^{\prime}$ to $\mathrm{KB}$ otherwise

11. No answer.

The output of the above algorithm is a combination of words.

\section{Result AND CONCLUSION}

The implementation of system is under processing. Tokenization and tagging of English statement are very important phases of the system.

The system is able to provide answer from a 1000-lines input provided in the form of a short story, write-up, etc.

The proposed reasoning algorithm that uses semantic net and script will be used for hybrid KR system and can be used in daily life activities as the system is capable of representing such knowledge. Combination of declarative and procedural 
techniques makes the system interactive and user friendly. This algorithm will be used for declarative as well as procedural knowledge. The reasoning algorithm can be utilized in many applications of AI and robotics. The proposed reasoning algorithm is used to infer the knowledge from the existing knowledge base. In its advanced stages of development, the proposed system can act as an intelligent system like a QAS.

\section{REFERENCES}

[1] Benjamin Cummings. Brewster, et al., , Knowledge representation with ontology's: the present and future, IEEE Intelligent Systems, pp. 72-81, 2004

[2] R. Davis, H. Shrobe, and P. Szolovits, "What is a Knowledge Representation?", AI Magazine, 14(1):17- 33, 1993

[3] Poonam Tanwar, Dr. T. V. Prasad and Dr. Kamlesh Datta, "Hybrid Technique for Effective Knowledge Representation", In "Advances in Intelligent Systems and Computing", Springer, Volume 178, 2012, pp 33-43.

[4] E. Rich and K. Knight, "Artificial Intelligence", 2nd Edition, McGrawHill, 1991.

[5] John F. Sowa, "Encyclopedia of Artificial Intelligence", Wiley, 2nd edition, 1992.

[6] Brachman $\mathrm{R}$ and Levesque $\mathrm{H}$, eds., "Readings in Knowledge Representation", Morgan Kaufman, 1985.

[7] Poonam Tanwar, T. V. Prasad and Kamlesh Datta, "An Effective Knowledge base system Architecture and Issues in Representation Techniques", Int. J. of Advancements in Tech. available at http://ijict.org/,2011.

[8] Poonam Tanwar, T. V. Prasad and Mahendra. S. Aswal, "Comparative Study of Three Declarative Knowledge Representation Techniques", Int. J. on Computer Sc. and Engg, Vol. 2, No. 7, 2010, pp. 2274-2281.

[9] Stuart Russell and Peter Norvig, Artificial Intelligence: A Modern Approach, 3rd Edition, Prentice Hall, 2009

[10] Morgenstern, "Knowledge Representation", Columbia University, 1999, available at http://wwwformal.stanford.edu/leora/krcourse/.

[11] Brachman and. Schmolze, "An overview of the KL-ONE Knowledge Representation System," Cognitive Science, Volume 9, Issue 2, Elsevier, pp 171-216, 1985.
[12] Tsai, Aoyama and Chang, "Rapid Prototyping using FRORL Language", Dept of Electrical Engg. and Computer Sc., Univ. of Illinois at Chicago, Chicago, IEEE, 1988.

[13] Tsai, Jaiig and Karen, Schellinger," RT-FRORL: A Formal Requirements Specification Language for Specifying Real-Time Systems", University of Illinois, Chicago, IEEE transaction, 1991.

[14] L William. Kuechler and Lim,Vaishnavi," A Smart Object Approach To Hybrid Knowledge Representation and Reasoning Strategies", Proc. of the 28th Annual Hawaii Int. Conf. on System Sciences, 1995.

[15] Shetty, Pierre Riccio, Quinqueton, "Extended Semantic Network for Knowledge Representation", Information Reuse and Integration, France, "IEEE-IRI, 2009.

[16] Singhe, Madur and Apperuma," Enhanced Frame-based Knowledge Representation for an Intelligent Environment", IEEE., KIMAS, Boston, USA, 2003.

[17] Poonam Tanwar, T.V. Prasad and Kamlesh Datta, "Hybrid Technique for Effective Knowledge Representation and a Comparative Study", Int. J. of Computer Sc. \& Engg Survey, Vol.3, No.4, pp 43-57, 2012

[18] Stillings, Luger, "Knowledge Representation", Chapters 4 and 5, 1994, available at www.hbcse.tifr.res.in/jrmcont/notespart1/node28.htm.

[19] Reena T. N. Shetty, Pierre-Michel Riccio and Joël Quinqueton,” Hybrid Model for Knowledge Representation", ICHIT '06 Proc. of the 2006 Int. Conf. on Hybrid Info. Tech., Vol. 01, 2006.

[20] Rathke, C., "Object-oriented Programming and Frame-based Knowledge Representation", 5th Int. Conf., Boston, 1993.

[21] Anne R, Diekema, et al., "What do you mean? Finding answer to complex Questions", National Aeronautics and Space Administration, New York.

[22] David Elworthy," Question Answering using a large NLP System", Microsoft Cambridge, Filtering Track, 2001.

[23] Mukul Aggarwal, "Information retrieval and Question answering NLP Approach: An Artificial Intelligence Application", Int. J. of Soft Computing \& Engg., June 2011.

[24] James Allen, George Ferguson, Daniel Gildea, Henry Kautz and Lenhart Schubert, "Artificial Intelligence, Natural Language Understanding, and Knowledge Representation and Reasoning", Natural Language Understanding, 2nd ed., Benjamin Cummings, 1994 